Review

\title{
VIRTUAL BRONCHOSCOPY, IMPORTANCE OF THE METHOD, APPLICATION AND PROSPECTS FOR TUMORS OF THE TRACHEA AND BRONCHI
}

\author{
M. A. Mitev ${ }^{1 *}$, N. Trajkova ${ }^{2}$, D. Arabadzhiev ${ }^{3}$, S. Valkanov $^{4}$, N. Georgieva ${ }^{5}$, \\ E. Obretenov ${ }^{6}$
}

${ }^{1}$ Department of Diagnostic Imaging, University Hospital "St. Kirkovich", Trakia University, Stara Zagora, Bulgaria

${ }^{2}$ Department of Diagnostic Imaging, University Hospital "St. George", Medical University, Plovdiv, Bulgaria

${ }^{3}$ Department of Pulmonology, University Hospital "St. Kirkovich", Trakia University, Stara Zagora, Bulgaria

${ }^{4}$ Department Surgery, Neurosurgery and Urology and Anesthesiology, Clinic of Neurosurgery, Trakia University, Stara Zagora, Bulgaria

${ }^{5}$ Department of Medical Physics, Biophysics, Roentgenology and Radiology, Trakia University, Stara Zagora, Bulgaria

${ }^{6}$ Department of Special Surgery / Thoracic Surgery, Vascular Surgery, Pediatric Surgery and Orthopedics and Traumatology / Clinic of Thoracic Surgery, Trakia University, Stara Zagora, Bulgaria

\begin{abstract}
Virtual bronchoscopy (VB) is 3-dimensional computer-generated technology, creating endobronchial images from spiral CT data. The study aims to present summarized results from researches of different foreign authors about the advantages of VB, the possibilities for its application and the effectiveness of its use in routine practice in the diagnosis of tumors of the trachea and bronchi. The three-dimensional model of the tracheobronchial tree allows assessment of the airways from inside. The majority of the examined studies relate to the diagnosis of new formations of the respiratory tract, preparation for surgery, assessment of the results of surgery, etc. VB is a noninvasive method allowing to examine the smallest bronchi. Relatively few studies in Bulgaria as well as the ensured during recent years new modern equipment for VB and MDCT, provide great opportunities for making VB examinations to study the effectiveness and its wide application in daily X-ray practice.
\end{abstract}

Key words: advantages, benign formations, effectiveness, tracheobronchial tree, Virtual bronchoscopy

\section{INTRODUCTION}

Virtual bronchoscopy (VB) is a new technique with computed tomography (CT) based images, which allows three-dimensional image (3D) and evaluation of the respiratory tract. The examination of the respiratory tract is carried out in a non-invasive manner, without further radiation exposure compared to the standard computed tomography of the thorax at patients with benign and malignant diseases, stenoses, etc., even at patients for whom the Fiberoptic bronchoscopy (FB) is too aggressive

\footnotetext{
*Correspondence to: Mitko Mitev, Department of Diagnostic Imaging, University Hospital "St. Kirkovich”, Trakia University - Stara Zagora, 6000 Mobile: +35988770 6079, E-mail: mitev.mitko69@gmail.com
}

or is not admissible. VB could be used to evaluate all levels of the respiratory tract from the upper airways to the periphery of the lung. It has important diagnostic, intervention and educational role. The study aims to present summarized results from researches of different authors about the advantages of VB, the possibilities for its application and the effectiveness of its use in routine practice in the diagnosis of tumors of the trachea and bronchi.

\section{RESULTS AND DISCUSSION}

Jones \& Athanasiou (1) determine VB as very discriminating in the evaluation of patients with significant stenoses of the respiratory tract as a result of the broad spectrum of pathological states. Gore et al. (2) confirm that VB is particularly suitable in some diseases of 
the chest as bronchoesophageal fistula, after lung transplantation, anastomoses, suspected aspiration of a foreign body and respiratory disabilities. VB enables accurately to inspect the lumen and the diameter of the bronchial tree, to assess the stenoses of the airways, to visualize remote prominent lesions. The images look very similar to what seen with FB. The intravenous introduction of contrast medium does not affect the quality of the VB images. Moreover, it is proven that VB can be useful for determining the appropriateness of the endobronchial procedures such as extensions, placement of a stent and laser ablation of endobronchial tumors. The main limitation of $\mathrm{VB}$ refers to the inability to reliably evaluate the mucosal surface of the airways. Therefore, VB may not be used for routine monitoring of patients at high risk of developing malignant diseases of the respiratory tract (3).

The examination of the patient's airway, the length of the lesion and the cross sectional area are important in the treatment of diseases of the respiratory system and usually require invasive endoscopy, which is considered the "gold standard" in this evaluation. VB is focused on the possibility of finding basic, central and lower airway abnormalities. These abnormalities may be:

(1) total blockage or partial stenoses caused by external compression from the tumor mass or from a foreign body;

(2) wall irregularities;

(3) mucosal or intraluminar prominence and partial local stenosis

(4) enlargement of the airways.

VB can be useful to visualize external compressions on the bronchial walls without the mucosa to be affected. These compressions can be caused by normal anatomical structures such as aortic arch, esophagus, etc. or be caused by abnormal structures such as external tumors, enlarged lymph nodes, fibrous masses, etc. The relationship between VB and axial CT and the multi-planar imaging usually allows to determine the exact localization and cause of the external pressures. However, small compressions are difficult to detect and can be underestimated at up to $25 \%$ of the patients. In most cases, VB is able to depict or refer to the tumor signs such as tumor mass, irregularity on the wall or cartilage loss. Also indirect signs such as stenosis or obstruction, compression or prominence often can be visualized. However, the cases of mucosal infiltration, vascular dilatation and necrosis are generally omitted.
MITEV M. A., et al. $V B$ in the diagnosis of central and peripheral carcinoma of the lung and trachea

The studies on the VB capabilities for diagnosis of cancer of the lung and trachea date back to the 90 s of the last century. Several studies show the feasibility and utility of VB at patients with primary or metastatic cancers involving the lungs and the mediastinum. Fleiter et al. (4) compare the results of the carried out FB and VB at patients with bronchial carcinoma, their capabilities and limitations. Diagnostic quality of $\mathrm{VB}$ is achieved in 19 of 20 patients. High-grade stenoses are revealed equally well with VB and FB. VB, according to the authors, offers the advantage to visualize areas, even distally, with high-grade stenoses. However, with VB discreet infiltration or extraluminal impression are not registered at 5 patients. At another patient, strong heart beats produced artificial impulse that prevented to assess the reconstruction. Liewald et al. (5) find that obstructive lesions at patients with lung cancer are diagnosed equally well with VB and with FB. Burke et al. (6) indicate that VB is precise in determining the width and length of the lesions fixed on the airways. RappBernhardt et al. (7) find $94 \%$ sensitivity and $99.7 \%$ specificity for evaluation of tracheobronchial stenoses due to bronchogenic carcinoma. According to another study of the same authors, statistical difference is not established in the location or the disposition of stenoses registered with $\mathrm{VB}$ or $\mathrm{FB}$ at patients with esophageal carcinoma. Hoppe et al. (8) find that VB increases the accuracy of the axial images from CT examinations related to the study of tracheal and bronchial stenoses. The authors find 98\% accuracy when performing VB to assess bronchial stenosis compared with CT only. They also show good correlation between VB and FB in assessing the degree of tracheobronchial stenoses resulting from endoluminal pathologies or from external signs. Finkelstein et al. (9) demonstrate sensitivity of $90 \%$ for endoluminal lesions, sensitivity of $100 \%$ for obstructive lesions, sensitivity of $16 \%$ for mucosal lesions, general sensitivity of $83 \%$ in patients with tracheobronchial malignancies, and 100\% specificity.

VB images, according to a number of authors, should be interpreted together with the axial CT images and the multi-planar reformatted images, if any. Combining the information from the VB images and CT images allows, according to them really to assess not only the intraluminal tumor spread, but also the extraluminal expansion of the mass and its 
relation with the bronchial tree. Other indicated advantage of VB is related to the fact that the bronchial tree can be assessed also distally to the stenoses that cannot be passed with FB $(10,4,5,11,6,12)$. In this way the length of the stenosis can be appreciated, which is not possible when the FB cannot pass the stenosis. Through the VB it is also possible to look at the distal border of endolumenal mass or stenosis with a "retroflexing" virtual bronchoscope. All these estimates show that VB could be a promising and noninvasive modality for identifying and classifying bronchial obstructions and endoluminal lesions. However, VB, according to a number of studies do not enable the detection of subtle lesions of the mucous membrane and therefore is not suitable for identification of premalignant lesions in the respiratory tract (13). Moreover, it is found that many viscous secretions in the bronchial tree or coagulated blood can mimic stenoses or occlusion and only careful comparisons with axial CT images provide the possibility for proper differentiation (14). Meyers and Mathisen (15) study the primary tracheal tumors as rare neoplasms, which are often characterized by non-painful and non-specific symptoms. Hoppe et al (8) apply multidetector CT VB to categorize tracheobronchial stenoses in patients with bronchial carcinoma and patient with non-respiratory diseases. The multidetector CT VB, according to the authors, is a reliable noninvasive method for accurate categorization of tracheobronchial stenoses. However they recommend the results to be interpreted with the axial $\mathrm{CT}$ images and the multi-planar converted images to evaluate the surrounding structures and for optimal spatial orientation. Finkelstein et al (16) present a comparative evaluation of the CT scans with ultra-high resolution and VB to detect tracheobronchial malignancies. They indicate that these new imaging techniques are valuable as additional conditions to provide information on FB, useful for the detection and management of malignant diseases of the respiratory tract. Joshi et al (17) conduct research on the role of multidetector CT for VB in assessing post-tracheostomies in tracheal stenoses. The authors study the technique and the utility of the VB as a tool for assessing the post-tracheostomy in tracheal stenoses and compare the results of VB and invasive bronchoscopy with a view to make further actions to treat lesions that were diagnosed. Galia et al (18) describe a single protocol for a single-layer spiral CT VB in the evaluation of patients with stenoses of the central airways and compare the results with a
MITEV M. A., et al.

FB. Jones et al. (1) define sensitivity of $84 \%$ and specificity of $75 \%$ from the applied VB, which indicate a good diagnostic work. Röttgen et al. (19) conduct research in order to compare the virtual with the flexible bronchoscopy to detect bronchial stenoses. Through the VB they detect 25 bronchial stenoses while with the flexible bronchoscopy they detect only 17 stenoses. Stenoses with a diameter of less than $50 \%$ they detect with VB 14 times and with flexible bronchoscopy 10 times. Stenoses with a diameter between 50 and $95 \%$ they detect 7 and 4 times, respectively, and barriers 4 and 3 times, respectively. They emphasize that the tracheobronchial stenoses are recognized with VB and even the virtual method enables highquality visualization of the stenoses and poststenotic areas that cannot be achieved with the FB. Shitrit et al. (20) compare the accuracy of VB with the FB and the pulmonary function testing (PFT) for the assessment of the tracheal stenosis and bronchial anastomotic stenosis. VB can be used to evaluate patients with some tracheobronchial stenoses after-treatment and thus to reduce the frequency of re-performing the invasive FB. Cuk et al. (21) publish results of VB and 3D spiral CT reconstructions applied at patients with lung cancer and subsequently treated with Syngo 3D software. According to the authors, the multilayer spiral $\mathrm{CT}$, generating $\mathrm{VB}$ is one of the latest developments in computer technology for three-dimensional visualization. They emphasize that VB allows non-invazive and fairly accurate assessment of the 3D tracheobronchial tree. Ferretti et al (22) indicate that tumors of the trachea and central bronchi can be benign and malignant as clinical picture, especially in benign tumors the picture can be confusing and they can be diagnosed as asthma or chronic bronchitis. They state that the radiography of the thorax has many limitations and often is not representative in patients with tumors of the central airways. They indicate MDCT as the most useful non-invasive method for the diagnosis and evaluation of neoplasms of the central airways.

Sundarakumar et al (23) conduct studies aiming to evaluate the diagnostic value of the $\mathrm{VB}$, the multi-planar reformatted images and the projection with minimal intensity in assessing airway stenoses. RadwanRöhrenschef and Burakowska (24) compare the results of the $\mathrm{VB}$ and the fibrobronchoscopy for the purposes of diagnosis when assessing central tumors of the lungs. The authors emphasize that VB is a new, noninvasive diagnostic method that allows 
MITEV M. A., et al.

visualization of the trachea and bronchi. In assessing bronchial stenoses they find sensitivity of $58.6 \%$ and specificity of $98.1 \%$, while in detecting the expansion of the carina they establish sensitivity of $60.7 \%$ and specificity of $97.7 \%$. On this basis, according to the authors, the results suggest that VB is a very sensitive and specific diagnostic method with high clinical importance in the evaluation of lung tumors with central location. Kotlayrov et al (12) evaluate the possibilities of the VB methodology in clarifying the diagnosis of the cancer diseases of the lungs and the tracheobronchial tree by comparing the data of the VB with those of the FB. According to the authors, the sensitivity and specificity of the VB in determining the tumor pathology of the trachea and the inner bronchial changes are respectively 91.5 and $90 \%$. They establish similar data for the actual Multidetector computed tomography (MDCT), $81 \%$ and $91 \%$ respectively. When combining the analyzes of the VB and the data of the MDCT according to the authors the sensitivity and specificity increase up to $95.3 \%$ and $96.5 \%$. The research team is convinced that the use of methods for obtaining the microstructures of the tracheobronchial tree by subsequent processing of the images and the images obtained through VB with minimal intensity, 3D images in a mode of darkened and semitransparent surfaces, allow to significantly extend the capabilities of the MDCT in determining, in diagnosis, the cancerous damages and the spread of the cancer in the lungs, bronchi and trachea. VB gives a clear idea of the nature of the spread of the tumor process. Luo et al (25) perform studies in order to evaluate the diagnostic value of the MDCT and its multiplanar reconstruction (MPR), volume rendering (VR) and $\mathrm{VB}$ as techniques for subsequent processing in cases of tumors of the trachea and the main bronchi.

VB is successfully applied for pre- and postoperative assessment of the bronchial tree. VB can provide accurate information for the luminal diameter and length of the stenosis, which is necessary and important for endobronchial procedures. VB also has an educational importance. In addition to the clinical indications, VB can be used for educational and research applications.

As a result of the performed review of some main studies, the following advantages and disadvantages of VB are identified:

\section{Advantages:}

- non-invasive procedure that can visualize areas inaccessible to the FB;

- short process without the need for contrasting;
- VB may be promising for identifying bronchial obstructions and for endoluminal lesions, as well as for assessment of the tracheobronchial tree beyond the stenosis;

- because the virtual bronchoscopic images are calculated from CT data, the information from the interior of the bronchial tree can be obtained together with the information from the outer side of the bronchial tree (CT);

- VB is accurate, but its accuracy is not $100 \%$ because there are cases of false positive and false negative results;

- It is expected that in the near future this technique will find its place in daily routine.

\section{Disadvantages:}

- VB does not allow detection of the subtle mucosal lesions.

- Because VB is an imaging technique based on CT, for CT it is always necessary for the patient to be exposed to radiation.

- VB depends on the availability and use of hardware, CT and software.

- VB will never replace FB, but in some cases it can help FB directly and sometimes it can give additional information.

\section{CONCLUSION}

The Virtual bronchoscopy with Multidetector $\mathrm{CT}$ is a modern method using $\mathrm{X}$-ray radiation. The prominent advantages of the Multidetector Virtual bronchoscopy with Multidetector computed tomograph as a noninvasive method that allows studying the smallest bronchi, precisely determining the location of pathological processes, the fastness of performance, the lack of contrasting etc. distinguish it as a modern reliable method for diagnosing lung and tracheal carcinomas, preand postoperative interventions and training, with opportunities for broad and effective implementation in real practice.

\section{REFERENCE}

1. Jones C. M, Athanasiou T. Is Virtual Bronchoscopy an Efficient Diagnostic Tool for the Thoracic Surgeon? The Annals of Thoracic Surgery; 79:365-374, 2005.

2. Gore M. A, Joshi A. R, Nagarajan G, Iyer S. P, Kulkarni T, Khandelwal A. Virtual bronchoscopy for diagnosis of inhalation injury in burnt patients. Burns; 30 (2):165168, 2004.

3. Wever W. De, Bogaert J, Verschakelen J. A. Virtual Bronchoscopy: Accuracy and Usefulness - An Overview. Semin Ultrasound CT MRI; 26:364-373, 2005.

4. Fleiter T, Merkle E. M, Aschoff A. J, Lang G, Stein M, Görich J, Liewald F, Rilinger N, 
Sokiranski R. Comparison of Real-Time Virtual and Fiberoptic Bronchoscopy in Patients with Bronchial Carcinoma: Opportunities and Limitations. AJR; 169(6):1591-1595, 1997.

5. Liewald F, Lang G, Fleiter T, Sokiranski R, Halter G, Orend K. H. Comparison of virtual and fiberoptic bronchoscopy. Thorac Cardiovasc Surg; 46:361-364, 1998.

6. Burke A. J, Vining D. J, McGuirt W.F. Jr, Postma G, Browne J. D. Evaluation of airway obstruction using virtual endoscopy. Laryngoscope; 110:23-29, 2000.

7. Rapp-Bernhardt U, Welte T, Doehring W, Kropf S, Bernhardt T. M. Diagnostic potential of virtual bronchoscopy: advantages in comparison with axial CT slices, MPR and mIP? Eur Radiol; 10:981-988, 2000.

8. Hoppe H, Walder B, Sonnenschein M, Vock P, Dinkel H. Multidetector CT Virtual Bronchoscopy to Grade Tracheobronchial Stenosis. AJR; 178:1195-1200, 2002.

9. Finkelstein S. E, Summers R. M, Nguen D. M, Schrump D. S. Virtual bronchoscopy for evaluation of airway disease. Thorac Surg Clin; 14:79-86, 2004.

10. Summers R. M, Feng D. H, Holland S. M, Shelhamer J. H. Virtual bronchoscopy: segmentation metod for real-time display. Radiology; 200(3):857-862, 1996.

11. McAdams H. P, Goodman Ph. C, Kussin P. Virtual Bronchoscopy for Directing Transbronchial Needle Aspiration of Hilar and Mediastinal Lymph Nodes: A Pilot Study. AJR; 170(5):1361-1364, 1998.

12. Kotlayrov P. M, Temirhanov S. Z, Flerov K. E, Gombolevskii V. A, Chernychenko N. V, et al. Virtual bronchoscopy in the diagnosis of lung cancer, in the assessment of its spread and in monitoring of post-operative changes. $J$ RNCPP MS RF; 13:1999-7264, 2013.

13. Finkelstein S. E, Summers R. M, Nguyen D. M, Stewart J. H. T, Tretler J. A, Schrump D. S. Virtual bronchoscopy for evaluation of malignant tumors of the thorax. $J$ Thorac Cardiovasc Surg; 123:967-972, 2002.

14.Polverosi R, Vigo M, Baron S, Rossi G. Evaluation of tracheobronchial lesions with spiral CT: comparison between virtual endoscopy and bronchoscopy. Radiol Med (Torino);102:313-319, 2001.

15. Meyers B. F, Mathisen D. J. Management of Tracheal Neoplasms. The Oncologist; 2:245243, 1997.

16. Finkelstein S. E, Schrump D. S, Nguyen D. M, St. M. Hewitt, Kunst Tr. F, Summers R. M. Comparative Evaluation of Super HighResolution CT Scan and Virtual Bronchoscopy for the Detection of
MITEV M. A., et al. Tracheobronchial Malignancies. Chest J; 124 (5):1834-1840, 2003.

17. Joshi A. R, Khanna P. C, Merchant S. A, Khandelwal A, Agrawal N, Karnik N. D. Role of Multidetector CT Virtual Bronchoscopy in the Evaluation of Posttracheostomy Tracheal Stenosis - a Preliminary Study. Japi; 51, 871-876, 2003.

18. Galia M, Lo C. A, Midiri M, Bellia M, Bartolotta T. V, Cademartiri F, De Maria M, Lagalla R.Virtual bronchoscopy in patients with central endobronchial stenosing lesions. Technique optimisation with single slice spiral CT. Radiol Med; 108(1-2): 28-28, 2004.

19. Rottgen R, Schurmann D, Pinkernelle J, et al. Detection of airways stenoses: comparison of virtual and flexible bronchoscopy. Fortschr Röntgenstr;177:338-343, 2005.

20. Shitrit D, Valdsislav P, Grubstein A, Bendayan D, Cohen M, Kramer M. R. Accuracy of Virtual Bronchoscopy for Grading Tracheobronchial Stenosis: Correlation W lith Pulmonary Function Test and Fiberoptic Bronchoscopy. Chest J; 128(5):3545-3550, 2005.

21. Ćuk V, Belina St, Fureš R, Buković D, Lovrić D, Šešo I. Virtual Bronchoscopy and 3D Spiral CT Reconstructions in the Management of Patient with Bronchial Cancer - Our Experience with Syngo 3D Postprocessing Software. Coll Antropol; 31(1): 315-320, 2007.

22.Ferretti G. R, Bithigoffer C, Righini C. A, Arbib F, Lantuejoul S, Jankowski A. Imaging of Tumors of the Trachea and Central Bronchi. Thoracic Surgery Clinics; 20(1):3145, 2010.

23. Sundarakumar D. K, Bhalla A. S, Sharma R, Hari S, Guleria R, Khilnani G. Multidetector CT evaluation of central airways stenoses: Comparison of virtual bronchoscopy, minimal-intensity projection, and multiplanar reformatted images. $J$ Radiol Imaging; 21(3):191-194, 2011.

24. Radwan-Rhrenschef P, Burakowska B. Virtual bronchoscopy versus bronchofiberoscopy - a comparison of diagnostic value in assessment of central lung tumours. Pneumonologia $i$ Alergologia Polska; 80 (2):127-139, 2003.

25. Luo M, Duan C, Qiu J, Li W, Zhu D, Cai W. Diagnostic Value of Multidetector CT and Its Multiplanar Reformation, Volume Rendering and Virtual Bronchoscopy Postprocessing Techniques for Primary Trachea and Main Bronchus Tumors. PLoS ONE; 10(9):e0137329, 2015. 